\title{
Investigation and analysis of the fungal community structure in moldy Lonicerae japonicae Flos through ITS2 sequencing
}

\author{
Zhenying Liu ${ }^{1}$, Yan $\mathrm{Zhao}^{1,2}$, Lingna Wang ${ }^{1}$, Gaobin $\mathrm{Pu}^{1}$ and Yongqing Zhang ${ }^{1, *}$ \\ ${ }^{1}$ Key Lab of Chinese Medicine Resources Conservation, State Administration of Traditional Chinese Medicine of Shandong Province of \\ China, Shandong University of Traditional Chinese Medicine, Jinan, 250000, China \\ ${ }^{2}$ School of Pharmacy, Key Laboratory of Molecular Pharmacology and Drug Evaluation (Yantai University), Ministry of Education, \\ Collaborative Innovation Center of Advanced Drug Delivery System and Biotech Drugs in Universities of Shandong, Yantai University, \\ Yantai 264005, China
}

\begin{abstract}
Lonicerae japonicae Flos is a traditional Chinese medicine that has the effect is used for clearing away heat and detoxification. When stored improperly, this medicine is susceptible to mold growth, causing fungal contamination, reducing its safety and clinical efficacy. In order to understand the fungal contamination of Lonicerae japonicae Flos, we utilized the Illumina NovaSeq6000 platform to characterize fungal communities associated with five moldy Lonicerae japonicae Flos samples from warehouses in China via the sequencing of fungal internal transcribed spacer 2 regions. These analyses led us to identify 3 phyla, 5 classes, 7 orders, 9 families, 13 genera, and 20 species of fungi in these samples. Of these, Ascomycota was the dominant phylum, while Cysticomycetes, Ascomycetes, and Staphylococci were relatively dominant at the class level, and the most prevalent genera were Aspergillus, Penicillium, Xanthomonas, Microcystis, Talaromyces, and Erysiphe with relative abundance values of 79\%, 21\%, 94\%, 100\%, and 98\%. In summary, in the present study we conducted the ITS-based comprehensive characterization of fungal communities associated with Lonicerae japonicae Flos for the first time. This approach aims to facilitate the early detection of fungal contamination in Lonicerae japonicae Flos, providing a theoretical basis for the study of anti-mold technologies.
\end{abstract}

\section{Introduction}

As the use of traditional Chinese medicine (TCM) is steadily growing throughout the world, the incidence of quality control issues associated with the production of these compounds is also a growing concern. Mildew growth is one of the most common quality challenges to TCM, with associated fungal growth having the potential to lead to mycotoxin production. The growth of fungi on TCMs occurs frequently during the growth, harvesting, cleaning, transportation, and storage of these compounds if conditions are not properly controlled [1-3]. For example, one study of 14 Ziziphi Spinosae Semen obtained from nine different sites in China identified three fungal phyla [4], while another analysis of Taiwanese pharmaceutical plants detected aflatoxins in $35 \%$ of all analyzed samples [5]. In one study conducted in South Korea, total aflatoxin or aflatoxin $\mathrm{B}_{1}\left(\mathrm{AFB}_{1}\right)$ levels in 72 out of 700 herbal medicine samples were found to be above legal limits [6]. In addition, Aspergillus, Penicillium, and Fusarium can readily grow on seeds, fruits, leaves, and flowers, producing mycotoxins such as aflatoxins, ochratoxin A, etc. In line with results from a study by Sun et al. that unsafely elevated levels of $\mathrm{AFB}_{1}$ and zearalenone in Semen Coicis samples [7]. This frequent

* Corresponding author: zyq622003@126.com mycotoxin contamination by mycotoxins has been linked to the high-efficiency transfer of compounds such as ochratoxin A (OTA) from Astragalus propinquus root samples to extracts derived therefrom [8], with lotus seeds similarly being found to be frequently contaminated with aflatoxins, ochratoxin A, zearalenone, deoxynivalenol, fumonisins, T-2, and HT-2 toxins [9]. Fungi of the Eurotium, Rhizopus, Aspergillus, and Wallemia genera have been found to grow on betel nut seeds during storage [10]. Such growth is highly prevalent, with another recent study having detected aflatoxin contamination on $67 \%$ of samples [11], and with many prior reports having attested to the fungal contamination of TCM preparations [12-14]. Long-term exposure to these mycotoxins can have harmful effects including an increased risk of cancer development $[15,16]$.

As such, it is vital that the growth of fungi in TCM preparations be monitored and limited. In order to limit the content of mycotoxins in herbal medicines and to ensure their safety, certain limits and guidelines have been developed. The Microbial Limit Standards in 2015 in the Chinese Pharmacopoeia defined, for the first time, microbial limit standards for TCM decoction pieces and laid out guidelines for mycotoxin detection and microbial identification [17]. Korean mycotoxin limit standards have also recently helped to increase the safety and 
consistency of TCMs [18]. $\mathrm{AFB}_{1}$ and OTA are classified as group 1 and $2 \mathrm{~B}$ carcinogens, respectively, by the International Agency for Research on Cancer (IARC) [19]. However, most of these limits only apply to certain medicines containing fatty oils and volatile oils, omitting many medicines due to their non-comprehensive nature.

Lonicerae japonicae Flos (LJF) is a dried bud or flower of the Lonicera japonica Thunb plant in the honeysuckle family. Traditionally, LJF buds are used in TCM for detoxification and to treat a range of conditions including boils, fevers, scabies, and related maladies [20]. More recent studies have highlighted the pharmacological activity of LJF as an antibacterial, antiviral, and antiinflammatory compound, and it has been administered to patients in order to prevent conditions such as H1N1 influenza and hand-foot-and-mouth disease infections [21-22]. There is also evidence for the beneficial effects of this compound in the context of the current coronavirus pandemic of 2020 [23-27]. While in storage, LJF is susceptible to deterioration when exposed to inappropriate temperatures or humidity, with mildew growth and moth contamination being the primary challenges to appropriate LJF storage [28]. Mildew growth can be difficult to prevent during rainy or warm weather, and can have a significant adverse impact on medicinal efficacy and safety.

Traditionally, fungi were primarily identified through isolation and culture-based approaches that are timeconsuming and have a high risk of failing to identify certain fungi, thereby inaccurately characterizing fungal diversity in a given setting [29-30]. In contrast, recently developed high-throughput sequencing approaches can readily characterize all fungi present within a given sample based on internally transcribed spacer (ITS) sequencing. Owing to their conservation, these ITS regions can be used as de facto fungal barcodes, enabling researchers to disentangle which fungi are present in a given sample [31]. Such ITS-based sequencing strategies have been employed to characterize low abundance fungi in soil, sediment, and air filter samples [32-33], therefore providing new opportunities for the detection of fungal diversity in TCMs

The present study was therefore designed to evaluate the diverse fungal communities via use of the Illumina NovaSeq6000 platform and demonstrated the diversity of fungal contamination in moldy LJF to offer a scientific basis for the evaluation and monitoring the safety and quality of LJF.

\section{Materials and methods}

\subsection{Samples}

Five (S1 - S5) LJF samples were collected at random from different local warehouses. All samples were affected by varying degrees of mildew growth (Fig. 1).
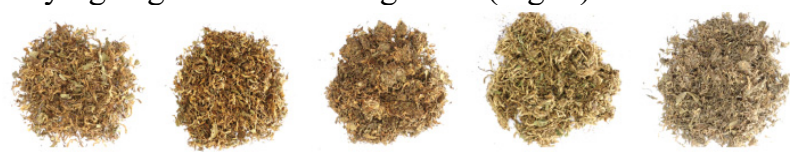

Figure 1. Five samples of moldy Lonicerae japonicae Flos.

\subsection{Genomic DNA extraction}

The CTAB method was used to extract genomic DNA (gDNA) from samples, after which sample purity and concentrations were assessed using 1\% agarose gels. All samples were then diluted to $1 \mathrm{ng} / \mu \mathrm{L}$ with sterile water.

\subsection{PCR amplification}

Each PCR reaction was conducted with a $30 \mu \mathrm{L}$ volume containing $15 \mu \mathrm{L}$ of Phusion ${ }^{\circledR}$ High-Fidelity PCR Master Mix (New England Biolabs); $0.2 \mu \mathrm{M}$ each of the ITS-1FF(5'-CTTGGTCATTTAGAGGAAGTAA-3') and ITS1F-R (5'-GCT GCGTTCTTCATCGATGC-3') primers, and $10 \mathrm{ng}$ of template DNA. Thermocycler settings were: $98^{\circ} \mathrm{Cfor} 1 \mathrm{~min} ; 30$ cycles of $98^{\circ} \mathrm{C}$ for $10 \mathrm{~s}, 50^{\circ} \mathrm{C}$ for $30 \mathrm{~s}$, and $72^{\circ} \mathrm{C}$ for $30 \mathrm{~s} ; 72^{\circ} \mathrm{C}$ for $5 \mathrm{~min}$.

\subsection{PCR product purification}

Equal volumes of all PCR products were mixed with $1 \mathrm{X}$ loading buffer containing SYBR Green followed by $2 \%$ agarose gel electrophoresis. Mixed PCR products were then purified using a GeneJET Gel Extraction Kit (Thermo Scientific).

\subsection{Library preparation and sequencing}

An Ion Plus Fragment Library Kit (Thermo Scientific) was used based on provided directions to prepare sequencing libraries. A Qubit@2.0 Fluorometer (Thermo Scientific) was used to assess library quality. Sequencing was then conducted with an Ion $\mathrm{S} 5^{\mathrm{TM}} \mathrm{XL}$ platform, generating $400 \mathrm{bp} / 600 \mathrm{bp}$ single-end reads.

\subsection{Data analysis}

Reads were assigned to individual samples based on unique barcode sequences, after which primer and barcode sequences were truncated. Raw reads were then filtered as appropriate yield high-quality cleaned reads with Cutadapt (v1.9.1, http://cutadapt. readthedocs.io /en/stable/). Reads were next compared to a Unite reference database (https://unite.ut.ee/) with the UCHIME algorithm

(http://www.drive5.com/usearch/manual/uchime_algo.ht $\mathrm{ml}$ ) to identify and eliminate chimeric sequences, yielding cleaned reads.

Uparse (v7.0.1001, http://drive5.com/uparse/) was used for sequence analyses, with OTU assignments being made based upon $a \geq 97 \%$ similarity cutoff. Representative OTU sequences were then subjected to further taxonomic annotation using the Unite Database (https://unite.ut.ee/) and the Blast algorithm with the QIIME software (v1.9.1)

(http://qiime.org/scripts/assign_taxonomy.html).

Phylogenetic relationships between OTUs were next compared via multiple sequence alignment with the MUSCLE software (v3.8.31, http:// www. drive 5.com/muscle/). OTU abundance information was normalized based upon the number of sequences in the 
sample containing the fewest sequences. Further alpha and beta diversity analyses were conducted using these normalized data.

Analyses of alpha diversity included Observed-species, Chao1, Shannon, Simpson, ACE, and Good's coverage indices that were calculated using QIIME (v1.7.0) and were visualized using $\mathrm{R}$ (v2.15.3). QIIME was additionally used for beta diversity analyses of weighted and unweighted unifrac distances between samples. PCA analyses were performed prior to cluster analyses in order to decrease the dimensionality of the input data. PCoA analyses were conducted based upon weighted or unweighted unifrac distances between samples. Samples were also hierarchically clustered based on unweighted unifrac by using an Unweighted Pair Group Method with Arithmetic Mean (UPGMA) approach.

\section{Results}

\subsection{Analysis of sample sequencing data}

Successful PCR amplification of the ITS2 sequences in all five samples was conducted. Sequencing of these samples yielded 422,098 total sequences and 400,465 clear reads between 230 and $250 \mathrm{bp}$ in length. The sample with the most reads (sample 2) was associated with 87,687 reads, while the sample with the fewest reads (sample 1) yielded 81,118 reads.

\subsection{High-throughput sequencing data analysis}

\subsubsection{OTU analysis}

We began by surveying the fungal communities in these samples through OTU clustering at a 97\% identity threshold, with representative OTU sequences being annotated. Following cluster analyses, these sequences were grouped into 317 different OTUs. Sample normalization was then conducted, and a petal diagram was used to identify common and unique OTUs in these different samples (Fig. 2). These results indicated that sample 4 exhibited the greatest number of unique OTUs and the greatest diversity. A total of 46 OTUs were detected in all five samples.

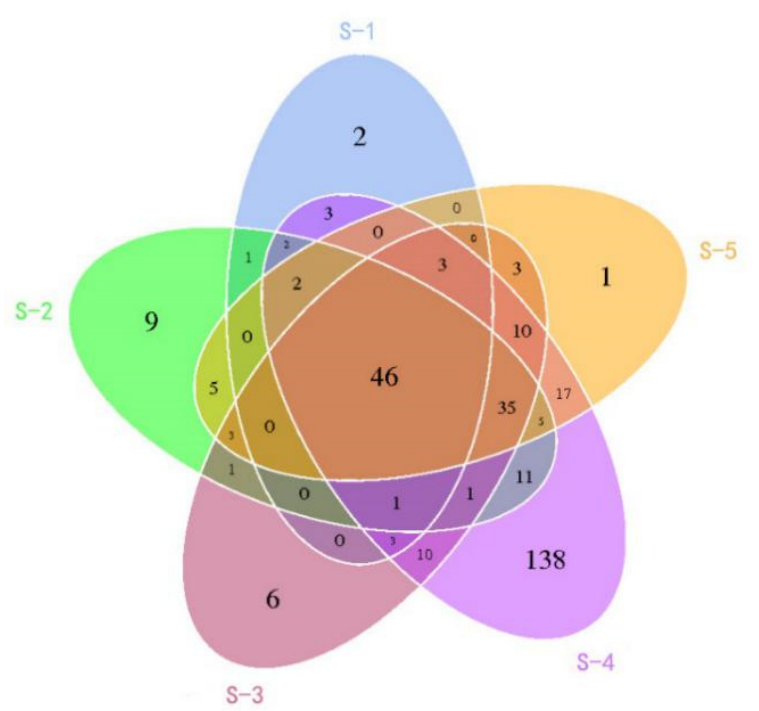

Figure 2. Petal diagram of operational taxonomic units (OTUs) in the moldy Lonicerae japonicae Flos. Each circle in the figure represents a sample, the number of circles and overlapping circles represents the number of OTUs shared between the samples, and the number without overlap represents the number of unique OTUs of the sample.

To rationally determine whether our data were representative of true fungal diversity in these LJF samples and to directly reflect the rationality of sequencing data, we next constructed rarefaction curve based on the number of OTUs associated with a randomly sampled subset of these data (Fig. 3a). The resultant curves for all 5 samples were relatively flat, suggesting that sufficient sequencing has been conducted.

OTUs in each sample were next sorted based on relative abundance, with a rank abundance curve being drawn by graphing OTU relative abundance against sorted rank (Fig. 3b). Sample 4 exhibited the greatest fungal richness in these graphs, whereas samples 2,3 , and 5 exhibited relatively smooth curves suggesting the presence of a relatively uniform fungal distribution. Furthermore, the sample 1 curve exhibited a sharp drop at the beginning, suggesting that dominant flora was present within this sample.

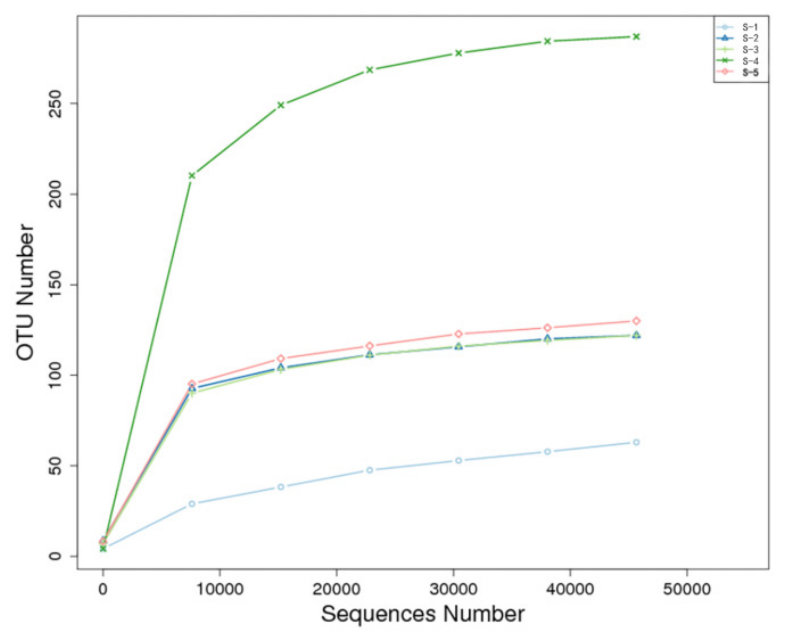




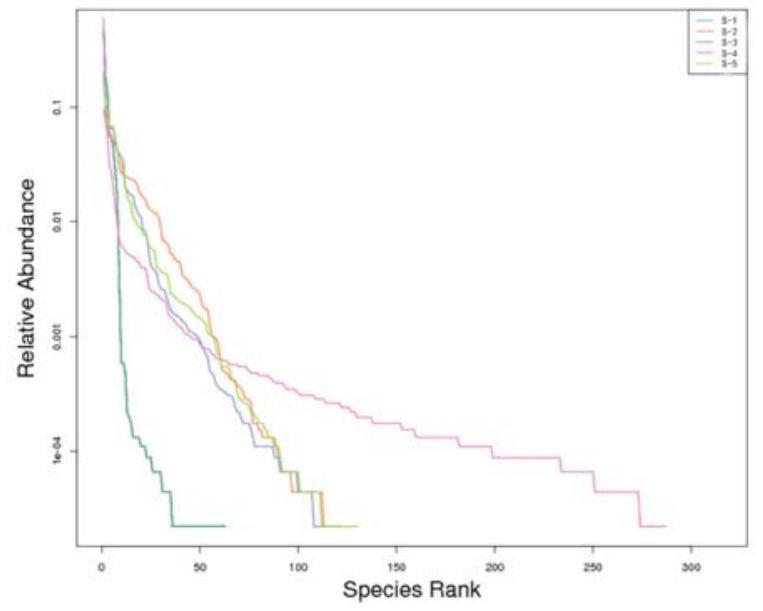

Figure 3. Curve of sample diversity within the group. (a) The dilution curve: the abscissa is the number of sequencing strips randomly selected from a sample, and the ordinate is the number of OTUs that can be constructed based on the number of sequencing strips, which is used to reflect the sequencing depth. Different samples are represented by curves of different colors; (b) The Rank Abundance curve: the abscissa is the serial number sorted by the abundance of OTUs, and the ordinate is the relative abundance of the corresponding OTUs.
Different samples are represented by polyline of different colors.

\subsubsection{Analyses of the diversity of fungal communities}

Alpha Diversity ( $\alpha$-diversity) was employed as a means of evaluating within-sample fungal diversity based on a number of different indices such as the Shannon, Simpson, chao1, ACE, and Good's coverage indices (Table 1). Species diversity was measured using the Shannon and Simpson indices, with higher Shannon index values and lower Simpson index values both corresponding to greater community diversity. In contrast, the ACE and Chaol indices were directly proportional to the number of different species in a given sample, whereas Good's coverage index was used to assess sampling completeness at a given sampling level.

Table 1. Five moldy Lonicerae japonicae Flos samples Alpha indices table.

\begin{tabular}{cccccc}
\hline sample name & shannon & simpson & chao1 & ACE & Good's coverage \\
\hline S-1 & 2.330 & 0.713 & 126.000 & 105.545 & 0.999 \\
S-2 & 5.146 & 0.960 & 124.647 & 128.033 & 1.000 \\
S-3 & 4.405 & 0.922 & 133.667 & 131.969 & 1.000 \\
S-4 & 3.045 & 0.626 & 290.792 & 291.074 & 1.000 \\
S-5 & 4.523 & 0.927 & 144.250 & 147.785 & 1.000 \\
\hline
\end{tabular}

As shown in Table 1, sample 2 exhibited the largest Shannon index value, whereas sample 4 exhibited the smallest Simpson index value, suggesting that these two samples exhibited the greatest fungal community diversity. Chaol and ACE indices further revealed that sample 1 contained the fewest identified species, whereas sample 4 contained significantly more species relative to these other samples. Good's coverage index for these five samples was $>99.9 \%$, thus indicating that a good overall sampling level had been achieved.

Beta Diversity ( $\beta$-diversity) was additionally employed as a means of evaluating differences in fungal communities between samples. Both weighted and unweighted unifrac distance indices were employed to measure dissimilarity coefficients between samples, with higher values corresponding to greater differences in fungal diversity between a given pair of samples. These weighted and unweighted unifrac distances between samples were used to draw heatmaps (Fig. 4), revealing that samples 2 and 5 were the most similar to one another. In contrast, samples 1 and 4 were significantly different from one another, in line with the above results demonstrating that these samples exhibited clear differences in overall species richness. We next clustered these samples using a sample UPGMA clustering tree approach (Fig. 5), Samples 5 and 2 were relatively close to one another in these plots, suggesting that the overall species composition of these two samples was similar.

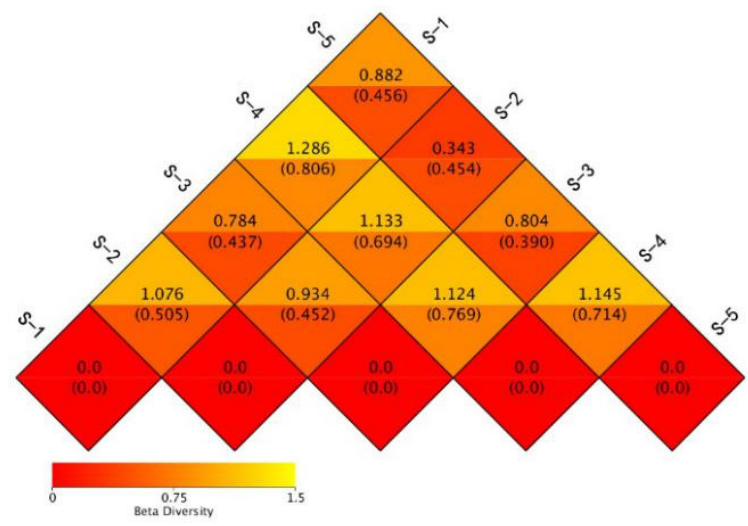

Figure 4. Beta Diversity index heatmap. The numbers in the box in the figure are the coefficients of dissimilarity between the samples.The smaller the difference coefficient, the smaller the difference in species diversity; In the same cell, the upper and lower values represent the distance of Weighted Unifrac and Unweighted Unifrac, respectively. 


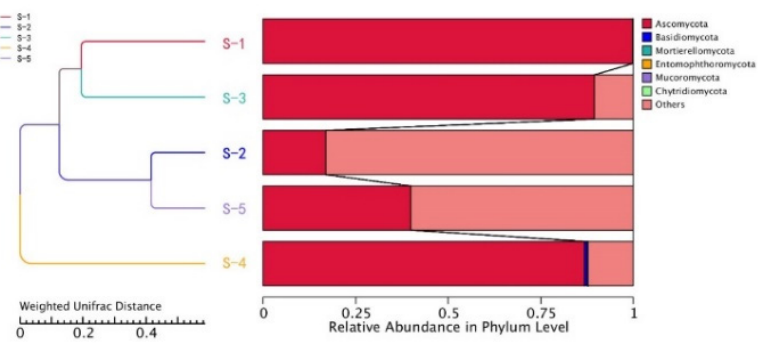

Figure 5. UPGMA clustering tree based on Un Weighted Unifrac distance. On the left is the UPGMA cluster tree structure, and on the right is the relative abundance distribution map of the samples at the gate level.

\subsection{Analyses of fungal community composition}

In total we identified 3 phyla, 5 classes, 7 order, 9 families, 13 genera, and 20 species of fungi in these tested samples. Ascomycota was the dominant phylum represented in these samples, with all other phyla being relatively rare, accounting for $100 \%, 17 \%, 90 \%, 87 \%$, and $40 \%$ of fungi in samples 1-5, respectively (Fig. 6a). Ascomycota members are known to cause certain pathogenic conditions including leaf spot, root rot, stem rot, branch wilding, and fruit rot. As such, we can hypothesize that these Ascomycota species were likely present on these LJF samples prior to harvesting. In contrast, the Basidiomycota and Zygomycota phyla were relatively rare in these samples, accounting for just $0.9 \%$ and $0.04 \%$ of identified fungi, respectively. At the class level, Eurotiomycetes, Dothideomycetes, and Tremellomvcetes were most dominant in these samples, while at the genera level Aspergillus, Penicillium, Xanthomonas, Microcystis, Talaromyces, and Erysiphe were most prevalent, with relative abundances of $79 \%, 21 \%, 94 \%, 100 \%$, and $98 \%$ in samples $1-5$, respectively. Clear differences in fungal community structure at the species levels were identified among these samples (Fig. 6b). For example, Aspergillus chevalieri, Aspergillus creber, Aspergillus niger, Aspergillus pseudoglaucus, and Xerochrysium dermatitidis were detected in all five samples, whereas other species were unique to individual samples.

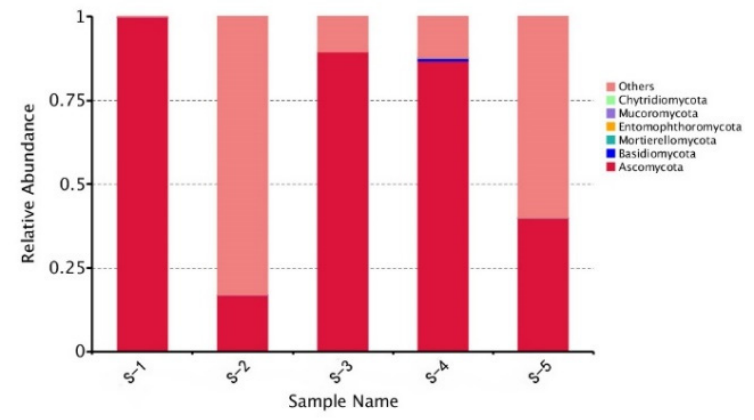

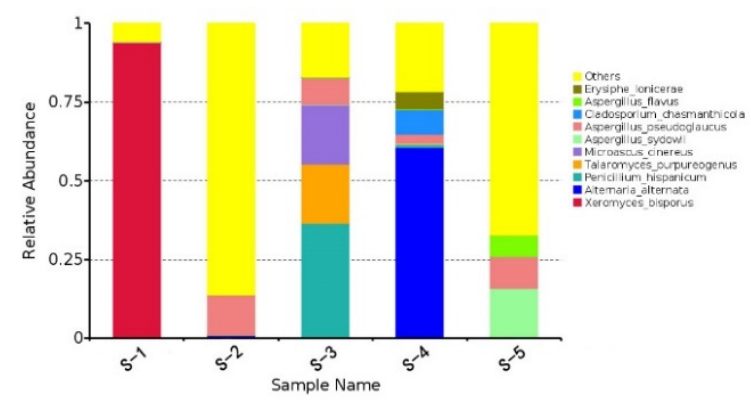

Figure 6. (a) Histogram of species relative abundance at the Phylum level. The abscissa is the sample name; the ordinate represents the relative abundance; Others represents the sum of the relative abundances of all the Phylums except the 10 Phylums in the figure.(b) species relative abundance at the species level.

No aflatoxin- or mycotoxin-producing fungi were found to be associated with the LJF samples analyzed in the present study. Aflatoxins are extremely dangerous and can be produced through interactions between Aspergillus flavus and Aspergillus parasiticus. While Aspergillus flavus was detectable in sample 5, no Aspergillus parasiticus was found in this or any other sample, thus precluding the possibility of aflatoxin production.

\section{Discussion}

\subsection{ITS sequencing-based characterization of fungal diversity in herbal medicine samples}

The ITS1 and ITS2 sequences are variable spacer regions between the highly conserved fungal $18 \mathrm{~S}, 5.8 \mathrm{~S}$, and $28 \mathrm{~S}$ rRNA genes. As these ITS regions are subjected to less selective pressure, they are relatively polymorphic such that while ITS sequences for a given species are relatively similar to one another, there are clear differences in these sequences between species. Owing to these properties, ITS sequences can serve as a catalog to gauge eukaryotic species diversity, and ITS sequencing is thus commonly used for phylogenetic analyses aimed at identifying fungal species associated with different herbal medicines.

Several prior studies have employed ITS-based approaches to analyzing herbal medicine samples. For example, ITS2 sequencing of Aconitum carmichaelii (Fuzi) root samples identified Phoma adonidicola as a putative cause of root rot, with Mortierella chlamydospora and Penicillium simplicissimum being predicted to further impair root health [30]. The ITS2 technology was utilized to reveal that Aspergillus flavus was also found to make up $36.29 \%$ of the total fungi present on the surface of Ziziphi Spinosae Semen [4]. An ITS-based approach to analyzing Areca nut seeds in storage revealed that fungal community diversity decreased during storage [34]. Furthermore, Gao et al. employed this technology in order to characterize 12 medicinal Dendrobium plants [35]. ITS sequencing can also be employed for phylogenetic and genetic evolutionary analyses of medicinal plants [36], for characterizing the fungi present within rhizosphere soils and non-medicinal portions of these medicinal plants[37], 
and for accurately identifying impure and counterfeit TCM preparations[38-39]. These approaches are thus invaluable as a means of ensuring TCM safety and efficacy.

With respect to LJF samples, ITS sequencing can be used not only to identify certain pathogens of pests and diseases including Helicoverpa armigera Hubner and Heterolocha jinyinhuaphaga Chu, but also to assess overall fungal community diversity in non-medicinal portions of these plants. By better understanding the fungal communities present in niches soil, leaf, and stem, it may be possible to more effectively control honeysuckle disease and other sources of damage. The present study was the first to employ an ITS sequencing approach in order to survey fungal community diversity in moldy LJF samples. Future analyses have the potential to leverage this technology in order to assess dynamic changes in mildew growth over time during LJF storage and to better understanding the differences in fungal communities associated with moldy and non-moldy samples.

\subsection{The status of LJF fungal diversity research}

Studies of LJF fungal diversity are primarily focused on identifying and utilizing endophytic and pathogenic fungi associated with these samples. Zhang et al. found $P$. pseudomycoides to be the causative agent responsible for honeysuckle brown spot disease, and also found these samples of LJF to be associated with fungi including Alternaria spp., Gentidocladium spp., Penicillium spp., Mucor spp., and Aspergillus spp [40]. Li et al. similarly isolated 38 strains of endophytic fungi from Henan LJF samples, with two of these fungi having been found to exhibit robust antibacterial activity against E. coli and Staphylococcus aureus [41]. Given that LJF grows during different periods in specific geographical regions, LJF samples are often associated with region-specific endophytic fungi that exhibit distinct biological activities. For example, one study of the endophytic fungi associated with Zhanjiang LJF revealed that Trichoderma pseudospora were the primary fungi present in floral samples from these plants, whereas distinct fungi were associated with plant stems and leaves [42]. Cao et al. found that vesicle-arbuscular mycorrhizal fungus can infect the roots of up to $81 \%$ of plants during the LJF growing season [37]. Another qualitative analysis of 80 isolated endophytic fungi associated with samples from Henan and Xinmi detected multiple strains capable of actively producing chlorogenic acid [43]. Certain other studies have also been found that fungi associated with LJF plants can exhibit medicinal properties capable of treating certain diseases [44].

Mold samples examined in the present study revealed the presence of endophytic fungi including Penicillium and Aspergillus, consistent with some of the above studies. In addition, Erysiphe mildew fungi were detected in these samples. As these samples had been stored in a range of environments, they exhibited variable fungal community diversity, although Aspergillus species were detected in all five samples. Future studies of endophytic and pathogenic fungi present within LJF samples will be necessary in order to better prevent and control the growth of damaging microbes on these TCMs.

\subsection{Prevalence of mildew affects the quality of TCMs}

Improper harvesting, processing, storage, or transportation of TCMs compounds can lead to changes in their physical or chemical properties. Mildew growth is a common phenomenon that can adversely impact TCMs quality, potentially leading to the breakdown of certain oils or the de novo production of carcinogenic compounds.

Mildew contamination of TCMs can result in organic matter decomposition as a result of fungal metabolic activity, leading to decreases in active ingredient abundance. In a study conducted by Qin et al., for example, total alkaloid levels in Evodia herbal preparations were significantly lower in mold-contaminated samples than in non-contaminated samples [45]. There is also clear evidence that mildew growth can change active ingredient levels within TCMs, as demonstrated in a recent study of volatile oil contents in Myristica fragrans Houtt. during different stages of mildew growth [46]. Similarly, mildew growth on Angelica sinesis preparations has been shown to be associated with $60.71 \%$ and $53.16 \%$ reductions in ferulic acid and ligustilide contents, potentially adversely affecting clinical efficacy [47]. Tanshinone compound levels in Salvia miltiorrhiza were also found to decrease as a function of mildew growth, whereas such growth was associated with increases in salvianolic acid levels in these same samples [48].

Furthermore, certain TCM compounds rich in fatty oils such as Armeniacae Semen Amarum, Platycladi Semen, Pruni Semen, and Presicae Semen can undergo material decomposition following mold growth, leading to internal changes that can alter fat or sugar levels, thus causing medicines to become oily or sticky. Moldy TCMs can lose their efficacy and/or become carcinogenic. Indeed, contamination with Aspergillus flavus-derived carcinogenic aflatoxins can be extremely harmful. Following mildew growth, other TCMs such as Hordei Fructus Germinatus, Nelumbinis Semen, and Myristicae Semen have similarly been found to be contaminated with aflatoxin, fumonisin, and ochratoxin [49]. Comparable findings have also been observed for kudzu root [50] and other samples.

The mildew-contaminated LJF samples used in the present study appeared dark, gray, and dry, in clear contrast to normal LJF samples. Other mildewed LJF samples may appear turquoise in color and have a musty smell. We hypothesize that mildew growth may affect levels of chlorogenic acid, isochlorogenic acid, and luteolin in LJF, thereby affecting its pharmacological activity. Further research will be needed, however, to determine the degree to which individual fungi affect this phenotype. Few studies to date have characterized such contaminated LJF samples, and the present article is the first to our knowledge to have defined the fungal communities present in these samples. In future studies, we will evaluate chlorogenic acid contents in these contaminated LJF samples while also evaluating their quality and pharmacological potency. It is vital that LJF 
be prepared and stored under dry, well-ventilated conditions in order to minimize the risk of fungal growth and to thereby preserve the quality and safety of this important herbal medicine.

\section{Reference}

1. M. Jalili, S. Jinap, Food Control, 24, 160-164(2012).

2. A. M. Katsurayama, L. M. Martins, B. T. Iamanaka, M. H. P. Fungaro, J. J. Silva, J. C. Frisvad, J. I. Pitt, M. H. Taniwaki, Int J Food Microbiol, 266, 213221(2018).

3. T. Stevic, S. Pavlovic, S. Stankovic, K. Savikin, Arch Biol Sci, 64, 49-58(2012).

4. M. Guo, W. Jiang, J. Luo, M. Yang, X. Pang, Toxins, 10, 494(2018).

5. M. Chien, C. Yang, C. Huang, C. Chen, J Food Drug Anal, 26, 1154-1159(2018).

6. W.B. Shim, K. Kim, J.A. Ofori, Y.C. Chung, D.H. Chung, J Food Prot, 75, 1991-1999(2012).

7. S. Sun, P. Zheng, S. Zhao, H. Liu, Z. Wang, T. Peng, J. Wang, K. Yao, S. Wang, Y. Zeng, H. Jiang, Food Addit Contam A, 35, 2434-2442(2018).

8. J. Toman, V. Ostry, Y. Grosse, T. Roubal, F. Malir, Mycotoxin Res, 34, 223-227(2018).

9. P. Huang, W. Kong, S. Wang, R. Wang, J. Lu, M. Yang, J Pharm Pharmacol, 70, 1378-1388(2018).

10. X. Jiang, H. Li, J. Xiong, J. Deng, H. Xu, L. Yao, X. Luo, Chinese Journal of Food Science ,19, 238243(2019).

11. N. Wang, C. Duan, S. Li, X. Geng, K. Ding, Y. Guan, J Chromatogra, 460894(2020).

12. L. Chen, W. Guo, Y. Zheng, J. Zhou, T. Liu, W. Chen, D. Liang, M. Zhao, Y. Zhu, Q. Wu, J. Zhang, Toxins, 12, 30(2020).

13. T. Li, D. Jiang, X. H, X. Chang, Z. Xu, Y. Gong, G. Hua, C. Liu, Acta Pharm Sinica, 1- 12(2019).

14. Q. Li, Y. Fan, M. Song, F. Qin, M. Yang, J Pharm Anal, 39, 1945-1953(2019).

15. S.M. Roberts, R.C. James, P.L. Williams, Principles of Toxicology: Environmental and Industrial Applications, 2nd ed; John Wiley \& Sons: Hoboken, NJ, USA,2000(2015).

16. S. Marin, A.J. Ramos, G. Cano-Sancho, V. Sanchis, Food Chem Toxicol, 60, 218-237(2013).

17. Chinese Pharmacopeia Commission. Pharmacopoeia of People's Republic of China (Part 4); Press of Chemical Industry: Beijing, China, esepp(2015).

18. X. Jun, The number of species restricted in Korea for mycotoxin has been increased to 19 in South Korea. International Reference of TCM,11, 8-8(2008).

19. IARC. In IARC Monographs on the Evaluation of Carcinogenic Risks to Humans; International Agency for Research on Cancer: Lyon, France, 56(1993).

20. Chinese Pharmacopeia Commission. Pharmacopoeia of People's Republic of China (Part 1); Press of
Chemical Industry: Beijing, China, esepp. 247248(2015).

21. B. Yang, Z. Zhong, T. Wang, Y. Ou, J. Tian, S. Komatsu, L. Zhang, J Proteomics, 208, 103470(2019).

22. Y. Yuan, L. Qi, J. Yu, X. Wang, L. Huang, Int J Mol Sci, 16, 521-534(2014).

23. Y. Jin, L. Cai, Z. Cheng, H. Cheng, T. Deng, Y. Fan, C. Fang, D. Huang, L. Huang, Y. Han, B. Hu, et al. Mil Med Res, 7,4(2020).

24. H. Lu, Biosci Trends, 14, 69-71(2020).

25. Z. Chen, T. Nakamura, Phytother Res, 18, 5924(2004).

26. Z. Wang, X. Chen, Y. Lu, F. Chen, W. Zhang, Biosci Trends, 14, 64-68(2020).

27. D. Zhang, K. Wu, X. Zhang, S. Deng, B. Peng, J Integr Med, 18, 152-158(2020).

28. M. Li, Henan University(2019).

29. E.M. Mateo, J.V. Gomez, N. Montoya, R. MateoCastro, J.V. Gimeno-Adelantado, M. Jimenez, A. Domenech-Carbo, Food Chem, 267, 91-100(2018).

30. W. Wang, D. Zhang, H. Wen, Q. Wang, C. Peng, J. Gao, PLoS One, 13, e205891(2018).

31. J. Xu, Fungal DNA Barcoding. National Research Council Canada(2016).

32. S. Lee, C. An, S. Xu, N. Yamamoto, Lett Appl Microbiol, 63, 165-171(2016).

33. Y. Lin, Y. Ye, Y. Hu, H. Shi, Ecotoxicol Environ Saf,180, 557-564(2019).

34. X. Jiang, H. Li, J. Xiong, J. Deng, H. Xu, L. Yao, X. Luo, Chinese Journal of Food Science, 19, 238243(2019).

35. J. Gao, J.L. Cai, J.W. Chen, S.Q. Xie, S.C. Yang, J.L. Zhang, Chinese Medicinal Materials, 42, 12551260(2019).

36. P. Guan, X. Liu, C.Q. Peng, P. Yang, X.Y. Chen, M.D. Li, J.M. Shi, Molecular plant breeding,17, 19581964(2019).

37. C. Cao, J. Zhang, X. Yang, Y. Shao, Observation of Northwest Plant Journal, 36, 479-485(2019).

38. J. Li, R. Zhang, S. Yu, X. Wang, X. Leng, L. Shi, Acta Pharmaceutica,54, 937-943(2019).

39. H. Wang, L. Shi, J. Zhou, G. Zhu, China Journal of Chinese Materia Medica, 43, 4055-4061(2018).

40. Y. Zhang; X. Liu, Z. Feng, J. Huang, J. Yang, G. Wang, H. Yang, Journal of Hebei Agricultural University,39, 73-77(2019).

41. J. Li, H. Zhang, N. Liu, X. Liu, L. Li, Chinese Journal of Antibiotics, 35, 236-237(2020).

42. H. Li, L. Yu, Guangdong Agricultural Sciences, 41, 75-78(2014).

43. M. Leng, C. Dong, Y. Qiao, H. Ye, Henan Agricultural Sciences, 43, 107-111(2014).

44. L. Xu, Y. Zhang, Plant Journal, 27(2002).

45. H.J. Qin, Y. Zhang, L. Ma, S.N. Chen, Anhui Medical and Pharmaceutical Journal, 17, 952-953(2013). 
46. R. Yang, J. Li, H. Chen, Y. Yu, H. Zou, Y. Yan, Chinese Journal of TCM, 34, 4812-4815(2019).

47. H. Yang, C. Chen, X. Zhang, Y. Liang, Guangzhou Chemical Industry, 47, 87-90(2019).

48. Z. Zhan, W. Fang, X. Ma, T. Chen, et.al, Chinese Medicine, 14,1(2019).

49. S. Wang, Research on the storage standard of easily medicinal herbs, Peking Union Medical College, Beijing, China(2016).

50. H. Zhou, W. Le, Shandong Journal of TCM,30, $197-$ 198(2011). 